\title{
A CHARACTERIZATION OF HILBERT SPACE
}

\author{
RONALD E. BRUCK, JR.
}

Abstract. A real Banach space $E$ of dimension $\geqq 3$ is an inner product space iff there exists a bounded smooth convex subset of $E$ which is the range of a nonexpansive retraction.

De Figueiredo and Karlovitz [3] have shown that if $E$ is a strictly convex real finite-dimensional Banach space and $\operatorname{dim} E \geqq 3$ then there can exist no bounded smooth nonexpansive retract of $E$ unless $E$ is a Hilbert space. (A subset $F$ of $E$ is a nonexpansive retract of $E$ if it is the range of a nonexpansive retraction $r: E \rightarrow F$.) This is a consequence of their more general result that if $E$ is reflexive and a convex nonexpansive retract of $E$ has at a boundary point $x_{0}$ a unique supporting hyperplane $x_{0}+H$ then $H$ is the range of a projection of norm 1 . As they have pointed out, the latter theorem fails in nonreflexive spaces (the unit ball of $C[0,1]$ furnishes a counterexample). Nevertheless, their first result is true in general:

THEOREM. Suppose $E$ is a real Banach space with $\operatorname{dim} E \geqq 3$. Then $E$ is an inner product space iff there exists a bounded smooth nonexpansive retract of $E$ with nonempty interior.

We separate out of the proof of the theorem a lemma, valid in all real Banach spaces:

Lemma. Suppose $F$ is a bounded smooth closed convex subset of a real Banach space $E$ and $F$ has nonempty interior. Then given disjoint bounded closed convex sets $M$ and $K$ in $E$ with $K$ compact, there exist $p \in E$ and $\lambda>0$ such that $K \subset p+\lambda F$ and $(p+\lambda F) \cap M=\varnothing$.

Proof of Lemma. Clearly the hypotheses and conclusions of the lemma are invariant if $K$ and $M$ are translated by the same vector; thus without loss of generality we may assume $0 \in K$. Similarly, we may also assume $0 \in$ int $F$. Since $K$ is compact and $M$ is closed, a basic separation theorem for convex sets assures the existence of a closed hyperplane $H$ which strictly separates $M$ and $K$; that is, there exist $w \in E^{*}, c \in R^{1}$

Received by the editors June 26, 1972 and, in revised form, August 21, 1973. AMS (MOS) subject classifications (1970). Primary 46C05.

Key words and phrases. Nonexpansive retract. 
such that $H=\{x \in E: w(x)=c\}$ and

$$
0 \leqq \sup \{w(y): y \in K\}<c<\inf \{w(y): y \in M\} .
$$

Since $K$ and $M$ are bounded, (1) also holds for functionals sufficiently close to $w$ (in the norm of $E^{*}$ ). By the Bishop-Phelps theorem [1] the support functionals of $F$ are dense in $E^{*}$; thus we may assume without loss of generality that the functional $w$ in (1) is a support functional of $F$.

If $w$ supports $F$ at $x_{0}$, then $H=\{x: w(x)=c\}$ is the tangent hyperplane to $\mu F$ at $\mu x_{0}$, where $\mu=c / w\left(x_{0}\right)>0$. Let $F_{t}=(1-t) \mu x_{0}+t \cdot \operatorname{int}(\mu F)$ for $t>0$. Since int $\mu F$ is convex and $\mu x_{0}$ is a boundary point of $\mu F$, it is easily seen that $F_{s} \subset F_{t}$ if $s<t$.

The family $\left\{F_{t}: t>0\right\}$ is an open cover of $K$. (In fact, since $\mu F$ is smooth at $\mu x_{0}$, it is easily verified that $\bigcup_{t>0} F_{t}$ is the open half-space $\{x: w(x)<c\}$ with boundary $H$ which includes int $(\mu F)$. By (1), $K$ is a subset of this open half-space.)

Since the cover $\left\{F_{t}: t>0\right\}$ is linearly ordered by inclusion and $K$ is compact, there exists $t>0$ such that $K \subset F_{t} \subset \mathrm{Cl}\left(F_{t}\right)$. On the other hand, $M \cap \mathrm{Cl}\left(F_{t}\right)=\varnothing$ because $M$ is a subset of the opposite open half-space $\{x: w(x)>c\}$. Since $\mathrm{Cl}\left(F_{t}\right)=(1-t) \mu x_{0}+\mu t F$, we may take $p=(1-t) \mu x_{0}$, $\lambda=\mu t$ to reach the conclusion of the lemma. Q.E.D.

Proof of Theorem. Necessity is trivial, since it is well known that the closed unit ball of a Hilbert space $E$ is a smooth nonexpansive retract of $E$. (In fact, every closed convex subset of $E$ is a nonexpansive retract of $E$-the proximity mapping is a nonexpansive retraction.)

To prove sufficiency, let $E_{1}$ be any three-dimensional subspace of $E$, $E_{0}$ any two-dimensional subspace of $E_{1}$, and $x_{0}$ any point of $E_{1} \backslash E_{0}$. Fix $R>0$ and define

$$
\begin{aligned}
K & =\left\{x \in E_{0}:\|x\| \leqq R\right\}, \\
M & =\left\{x \in E:\|x-y\| \leqq\left\|x_{0}-y\right\| \text { for all } y \in K\right\} .
\end{aligned}
$$

Then $K$ and $M$ are bounded closed convex sets with $K$ compact. We claim that $K \cap M \neq \varnothing$.

Otherwise, by the lemma there exist $p \in E$ and $\lambda>0$ such that $K \subset p+\lambda F$ and $(p+\lambda F) \cap M=\varnothing$. If $f$ is a nonexpansive retraction of $E$ onto $F$, it is easily verified that $g: x \mapsto \lambda f\left(\lambda^{-1}(x-p)\right)+p$ is a nonexpansive retraction of $E$ onto $p+\lambda F$. In particular, for any $y \in K \subset p+\lambda F$ we have $g(y)=y$ so

$$
\left\|g\left(x_{0}\right)-y\right\|=\left\|g\left(x_{0}\right)-g(y)\right\| \leqq\left\|x_{0}-y\right\| ;
$$

by definition, $g\left(x_{0}\right)$ therefore belongs to $M$. But $g\left(x_{0}\right) \in p+\lambda F$ since $g$ retracts $E$ onto $p+\lambda F$. This is a contradiction since $(p+\lambda F) \cap M=\varnothing$. 
We have shown that $K \cap M \neq \varnothing$ so that for each $R>0$ there exists $x_{R} \in E_{0}$ with

$$
\left\|x_{R}-y\right\| \leqq\left\|x_{0}-y\right\|
$$

for all $y$ in $E_{0}$ with $\|y\| \leqq R$. Since (2) holds for $y=0$ in particular, the set $\left\{x_{R}: R>0\right\}$ is bounded and (since $\operatorname{dim} E_{0}=2$ ) therefore relatively compact. Hence there exists a sequence $R_{n} \rightarrow \infty$ such that $x_{R_{n}} \rightarrow x_{\infty}$ for some $x_{\infty}$ in $E_{0}$. It follows from (2) that

$$
\left\|x_{\infty}-y\right\| \leqq\left\|x_{0}-y\right\| \quad \text { for all } y \in E_{0} .
$$

As in Kakutani [4], (3) implies the existence of a linear projection $P$ of $E_{1}$ onto $E_{0}$ with $\|P\|=1$.

To summarize: Whenever $E_{1}$ is a three-dimensional subspace of $E$ and $E_{0}$ is a two-dimensional subspace of $E_{1}$, then there exists a projection $P$ of $E_{1}$ onto $E_{0}$ with $\|P\|=1$. By Kakutani [4], $E_{1}$ must be an inner product space. Finally, since every three-dimensional subspace of $E$ is an inner product space, $E$ itself must be an inner product space. Q.E.D.

Remark. The same technique can be used to prove the following variant of Kakutani's theorem: If $E$ is a real Banach space of dimension $\geqq 3$ and every closed linear subspace of $E$ of codimension 1 is the range of a projection of norm 1 , then $E$ is an inner product space. It is only necessary to show that every closed half-space in $E$ is a nonexpansive retract of $E$; and this can be done as in Bruck [2, Theorem 5].

\section{REFERENCES}

1. E. Bishop and R. R. Phelps, The support functionals of convex sets, Proc. Sympos. Pure Math., vol. 7, Amer. Math. Soc., Providence, R.I., 1963, pp. 27-35. MR 27 \#4051.

2. R. E. Bruck, Jr., Nonexpansive projections on subsets of Banach spaces, Pacific J. Math. 47 (1973), 341-356.

3. D. G. de Figueiredo and L. A. Karlovitz, On the extension of contractions on normed spaces, Proc. Sympos. Pure Math., vol. 18, Part 1, Amer. Math. Soc., Providence, R.I., 1970, pp. 95-104. MR 43 \#877.

4. S. Kakutani, Some characterizations of Euclidean space, Japan J. Math. 16 (1939), 93-97. MR 1, 146.

Department of Mathematics, University of Southern California, Los Angeles, CALIFORNIA 90007 